Received: 14.06 .2020

Revised: 15.07 .2020

Accepted: 21.08 .2020

DOI: $10.17804 / 2410-9908.2020 .4 .035-047$

\title{
FRACTURE BEHAVIOR OF CELLULAR STRUCTURES OBTAINED BY SELECTIVE LASER MELTING
}

\author{
I. S. Kamantsev $^{1, \text { a)*}^{*}}$, Yu. N. Loginov ${ }^{2, \text { b) }}$, S. V. Belikov ${ }^{2,3, c)}$, S. I. Stepanov ${ }^{2, \text { d), }}$
} M. S. Karabanalov ${ }^{2, \text { e) }}$, and A. I. Golodnov ${ }^{2, \text { f) }}$

${ }^{1}$ Institute of Engineering Science, Ural Branch of the Russian Academy of Sciences, 34, Komsomolskaya St., Ekaterinburg, 620049, Russian Federation

${ }^{2}$ B.N. Yeltsin Ural Federal University,

19, Mira St., Ekaterinburg, 620002, Russian Federation

${ }^{3}$ M.N. Miheev Institute of Metal Physic, Ural Branch of the Russian Academy of Sciences, 18, S. Kovalevskoy St., Ekaterinburg, 620108, Russian Federation

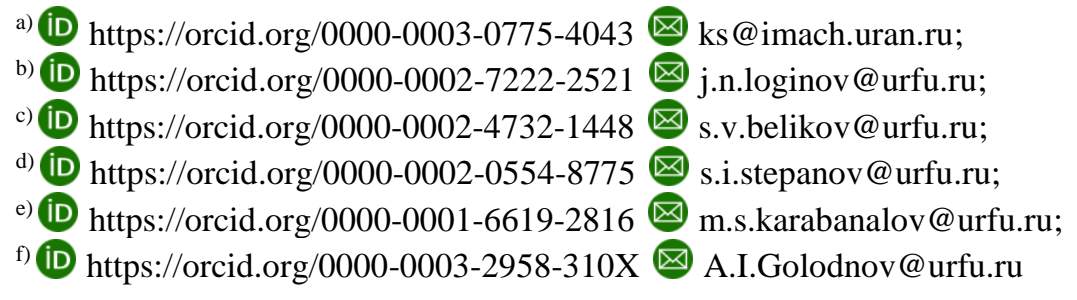

*Corresponding author. E-mail: ks@imach.uran.ru

Address for correspondence: ul. Komsomolskaya, 34, Ekaterinburg, 620049, Russian Federation Tel.: +7(343) 37447 25; fax: +7(343) 3745330

An example of samples with a cellular architecture, obtained by selective laser melting, is used to study the influence of the building direction of cellular objects on the characteristics of fracture under cyclic loading. The origin of their fracture has been revealed. The mechanism providing increased fatigue fracture resistance of objects which, along with the cellular structure, have anisotropy of properties due to the technological features of their production has been determined.

Keywords: selective laser melting, cellular structures, high-cycle fatigue, fracture.

\section{Acknowledgment}

This work was partially supported within the framework of the event "Creation and Functioning of a Network of International Scientific and Methodological Centers for the Expansion of the Best International Practices of Training, Retraining, and Internship of Advanced Digital Economy Personnel in the Fields of Mathematics, Informatics, and Technology" (Agreement No. 075-15-2019- 1907 dated 09.12.2019) and in accordance with the research plan for the IES UB RAS, theme AAAA-A18-118020790145-0. The equipment installed in the Plastometriya collective use center, IES UB RAS, was used in the experimental investigation.

\section{References}

1. Soro N., Attar H., Wu X., Dargusch M.S. Investigation of the structure and mechanical properties of additively manufactured Ti-6Al-4V biomedical scaffolds designed with a Schwartz primitive unit-cell. Materials Science and Engineering A, A 745, 2019, pp. 195-202. DOI: 10.1016/j.msea.2018.12.104. 
2. Golodnov A.I., Loginov Y.N., Stepanov S.I. Numeric loading simulation of titanium implant manufactured using 3d printing. Solid State Phenomena, vol. 284 SSP, 2018, pp. 380-385. DOI: $10.4028 /$ www.scientific.net/SSP.284.380.

3. Popov V.V., Muller-Kamskii G., Kovalevsky A., Kolomiets A., Ramon J. Design and 3D-printing of titanium bone implants: brief review of approach and clinical cases. Biomedical Engineering Letters, 2018, 8 (4), pp. 337-344. DOI: 10.1007/s13534-018-0080-5.

4. Van Hengel I.A.J., Gelderman F.S.A., Athanasiadis S., Minneboo M., Weinans H., Fluit A.C., Van der Eerden B.C.J., Fratila-Apachitei L.E., Apachitei I., Zadpoor A.A. Functionalitypacked additively manufactured porous titanium implants. Materials Today Bio, 2020, vol. 7. DOI: 10.1016/j.mtbio.2020.100060.

5. De Jonge C.P., Kolken H.M.A., Zadpoor A.A. Non-Auxetic Mechanical Metamaterials. Materials, 2019, 12 (4), 635. DOI: 10.3390/ma12040635.

6. Kilina P.N., Drozdov A.A., Sirotenko L.D. Formation samples with cellular structures by selective laser sintering of metal powders. Metalloobrabotka, 2015, no. 3 (87), pp. 29-31. (In Russian).

7. Cutolo A., Engelen B., Desmet W., Van Hooreweder B. Mechanical properties of diamond lattice Ti-6Al-4V structures produced by laser powder bed fusion: on the effect of the load direction. Journal of the Mechanical Behavior of Biomedical Materials, 2020, vol. 104, pp. 103656 (1-15). DOI: 10.1016/j.jmbbm.2020.103656.

8. $\quad$ Kazantseva N.V., Ezhov I.V., Vinogradova N.I., Il'inykh M.V., Fefelov A.S., Davydov D.I., Oleneva O.A. \& Karabanalov M.S. Effect of Built Geometry on the Microstructure and Strength Characteristics of the Ti-6Al-4V Alloy Prepared by the Selective Laser Melting. Phys. Metals Metallogr., 2018, no. 119, pp. 1079-1086. DOI: 10.1134/S0031918X18110066.

9. Cain V., Thijs L., Van Humbeeck J., Van Hooreweder B., Knutsen R. Crack propagation and fracture toughness of Ti6Al4V alloy produced by selective laser melting. Add. Man., 2015, vol. 5, pp. 68-76. DOI: 10.1016/j.addma.2014.12.006.

10. Kok Y., Tan X.P., Wang P., Nai M.L.S., Loh N.H., Liu E., Tor S.B. Anisotropy and heterogeneity of microstructure and mechanical properties in metal additive manufacturing: A critical review. Materials and Design, 2018, vol. 139, pp. 565-586. DOI: 10.1016/j.matdes.2017.11.021. Available at: https://www.sciencedirect.com/science/article/pii/S0264127517310493\#!

11. Hartunian P., Eshraghi M. Effect of Build Orientation on the Microstructure and Mechanical Properties of Selective Laser-Melted Ti-6Al-4V Alloy. J. Manuf. Mater. Process., 2018, 2 (4), 69. DOI: 10.3390/jmmp2040069.

12. Barba D., Alabort C., Tang Y.T., Viscasillas M.J., Reed R.C., Alabort E. On the size and orientation effect in additive manufactured Ti-6Al-4V. Materials and Design, 2020, vol. 186, 108235. DOI: https://doi.org/10.1016/j.matdes.2019.108235.

13. Stepanov S.I., Loginov Y.N., Kuznetsov V.P., Popov A.A. Effect of Annealing on the Structure and Properties of Titanium Alloy with Cellular Architecture for Medical Applications. Metal Science and Heat Treatment, 2018, 60 (5-6), pp. 315-321. DOI: 10.1007/s11041-018-0278-2.

14. Amin Yavari S., Ahmadi S.M., Pouran B., Schrooten J., Weinans H., Zadpoor A.A. Relationship between unit cell type and porosity and the fatigue behavior of selective laser melted metabiomaterials. Journal of the Mechanical Behavior of Biomedical Materials, 2015, vol. 43, pp. 91-100. DOI: 10.1016/j.jmbbm.2014.12.015.

15. Van Hooreweder B., Apers Y., Lietaert K., Kruth J.P. Improving the fatigue performance of porous metallic biomaterials produced by Selective Laser Melting. Acta Biomaterialia, 2017, vol. 47, pp. 193-202. DOI: 10.1016/j.actbio.2016.10.005.

16. Botvina L.R. Kinetika razrusheniya konstruktsionnykh materialov [Fracture Kinetics of Construction Materials]. Moscow, Nauka Publ., 1989, 230 p.

17. Kotsan'da S. Ustalostnoye rastreskivaniye metallov [Fatigue Cracking of Metals, transl. from Polish by G.N. Mekheda, S.Ya. Yarema, ed.]. Moscow, Metallurgiya Publ., 1990, 632 p. 
18. Volkov S.S. The Effect of Damage at An Ensemble of Microstructure Points on the Margin of Safety in Structurally Heterogeneous Materials // Diagnostics, Resource and Mechanics of materials and structures, 2019, iss. 5, pp. 60-72. DOI: 10.17804/2410-9908.2019.5.060-072. Available at: http://dream-journal.org/issues/2019-5/2019-5_274.html (accessed: 13.08.2020).

19. Loginov Y., Stepanov S., Khanykova C. Inhomogeneity of deformed state during compression testing of titanium implant. In: 2017 MATEC Web of Conferences, 13th International Scientific-Technical Conference on Dynamic of Technical Systems, DTS 2017, Rostov-on-Don, Sept. 13-15, 2017, vol. 132, 03009. DOI: 10.1051/matecconf/201713203009.

20. Maamoun A.H., Xue Y.F., Elbestawi M.A., Veldhuis S.C. Effect of Selective Laser Melting Process Parameters on the Quality of Al Alloy Parts: Powder Characterization, Density, Surface Roughness, and Dimensional Accuracy. Materials, 2018, 11 (12), 2343. DOI: 10.3390/ma11122343.

21. Gibson L. Ashby M. Cellular Solids: Structure and Properties, 2nd ed., Cambridge Solid State Science Series, Cambridge, Cambridge University Press, 1997. DOI: 10.1017/CBO9781139878326.

22. Xu Z.W., Liu A., Wang X.S. The influence of building direction on the fatigue crack propagation behavior of Ti6A14V alloy produced by selective laser melting. Materials Science and Engineering: A, 2019, vol. 767, 138409. DOI: 10.1016/j.msea.2019.138409. 
Подана в журнал: 14.06 .2020

УдК 620.168

DOI: $10.17804 / 2410-9908.2020 .4 .035-047$

\title{
ОСОБЕННОСТИ РАЗРУШЕНИЯ ЯЧЕИСТЫХ СТРУКТУР, ПОЛУЧЕННЫХ ЛАЗЕРНЫМ СЕЛЕКТИВНЫМ СПЛАВЛЕНИЕМ
}

\author{
И. С. Каманцев ${ }^{1, \text { a)* }}$, Ю. Н. Логинов ${ }^{2, \text { б) }}$, С. В. Беликов ${ }^{2,3, \text { в) С. И. Степанов }}{ }^{2, \text { г) }}$, \\ М. С. Карабаналов ${ }^{2, \text { д) }}$ А. И. Голоднов ${ }^{2, \text { e) }}$ \\ ${ }^{1}$ Институт машиноведения УрО РАН, г. Екатеринбург, Российская Федераиия \\ ${ }^{2}$ Уральский федеральный университет, г. Екатеринбург, Российская Федерация \\ ${ }^{3}$ Институт физики металлов им. М. Н. Михеева УрО РАН, г. Екатеринбург, Российская Федерация \\ a) iD https://orcid.org/0000-0003-0775-4043 ks@imach.uran.ru; \\ б) (iD https://orcid.org/0000-0002-7222-2521 $\otimes$ j.n.loginov@urfu.ru; \\ в) (DD https://orcid.org/0000-0002-4732-1448 s.v.belikov@urfu.ru; \\ r) (iD https://orcid.org/0000-0002-0554-8775 s.i.stepanov@urfu.ru; \\ д) (iD https://orcid.org/0000-0001-6619-2816 m.s.karabanalov@urfu.ru; \\ e) iD https://orcid.org/0000-0003-2958-310X ه A.I.Golodnov@urfu.ru \\ *Ответственный автор. Электронная почта: ks@imach.uran.ru \\ Адрес для переписки: ул. Комсомольская, 34, г. Екатеринбург, 620049, Российская Федерация \\ Тел.: +7(343) 374-47-25; факс: +7(343) 374-53-30
}

В работе на примере образцов с ячеистой архитектурой, полученных лазерным селективным сплавлением, рассмотрено влияние направления синтеза объектов ячеистой формы на характеристики разрушения в условиях циклического нагружения. Выявлены особенности в характере их разрушения и установлен механизм, обеспечивающий повышенное сопротивление усталостному разрушению объектов, имеющих наряду с ячеистым строением анизотропию свойств, обусловленных технологическими особенностями получения.

Ключевые слова: селективное лазерное сплавление, ячеистые структуры, многоцикловая усталость, разрушение.

\section{1. Введение}

Развитие технологии селективного лазерного сплавления (СЛС) позволяет создавать изделия со сложной пространственной геометрией, которые сложно, а иногда и невозможно изготовить традиционными методами литья, деформационной и механической обработки. Ярким примером является изготовление материалов с ячеистой структурой $[1,2]$. Изделия с подобными структурами нашли широкое применение в медицине [3, 4] и промышленности [5]. В настоящее время подробно изучено влияние технологических параметров на особенности формирования ячеистых структур $[1,6]$. Одним из наиболее значимых параметров при селективном сплавлении является ориентация модели относительно направления печати $[7,8]$. Для сплошных образцов выявлено влияние ориентации оси нагружения относительно направления печати образцов [9-13].

В то же время в известных работах по определению выносливости ячеистых структур внимания влиянию ориентации образца на долговечность $[14,15]$ и на сопротивление разрушению практически не уделяется. Особое внимание заслуживает изучение усталостных характеристик и особенностей сопротивления разрушению при циклических видах нагружения как наиболее опасных $[16,17]$. Выявленные на различных структурных уровнях закономерности позволят создать модели и цифровые двойники, учитывающие анизотропию свойств таких изделий, что в свою очередь облегчит прогнозирование ресурса [18]. В то же время, 
проведенные исследования ярко демонстрируют, что при разработке технологических процессов СЛС необходимо учитывать напряженно-деформированное состояние, возникающее при работе изделия [19].

Цель настоящей работы - выявление особенностей разрушения ячеистых образцов в зависимости от их ориентации относительно направления воздействия циклической нагрузки.

\section{2. Материал исследования}

Материалом исследований были выбраны ячеистые образцы прямоугольной формы. Модель ячеистой структуры и фотография боковой стороны образца представлены на рис. 1.

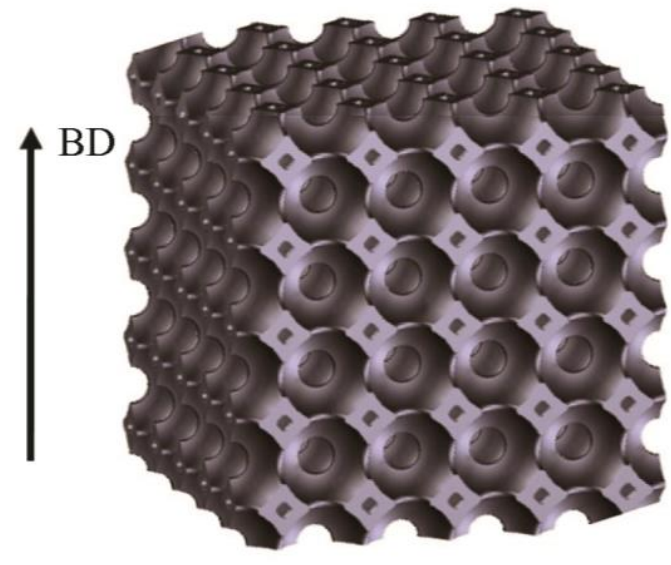

$a$

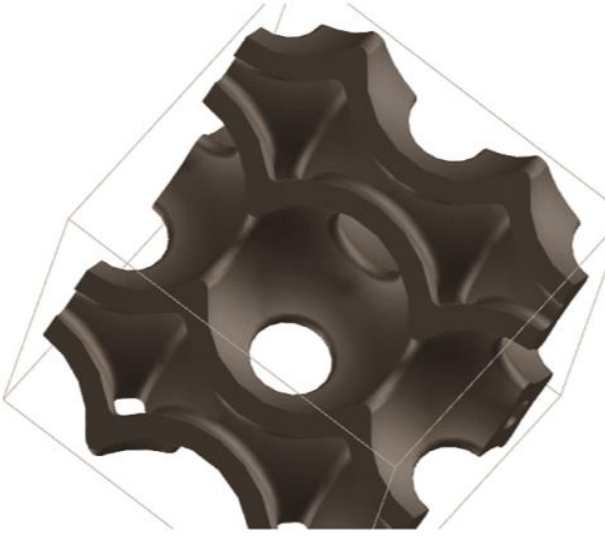

6
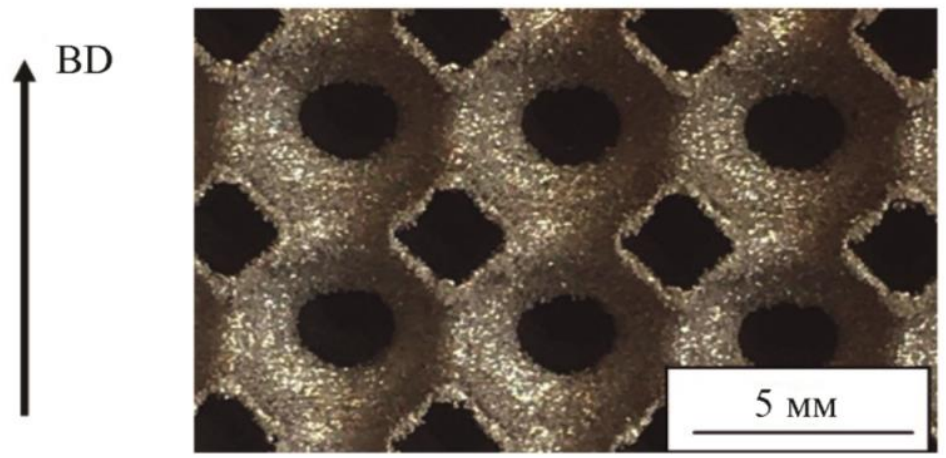

B

Рис. 1. Макет сетчатой структуры, состоящей из пересекающихся полых шаров $(a)$, и сегмент ячеистой структуры, демонстрирующий наличие каналов и отверстий между пересекающимися сферами (б); фото боковой стенки (в)

Ячеистая структура образцов выполнена в виде объемной решетки с расположением узлов на поверхности полых шаров, соединенных перемычками. Такая геометрия обеспечила относительную плотность (80 \%) на этапе электронной модели, которая может изменяться в результате особенностей процесса изготовления [20]. Можно отметить изменение формы нависающих стенок конструкции под собственным весом и изменение геометрии относительно электронной модели (рис. 1 в).

Образцы размером $20 \times 20 \times 20$ мм были получены методом селективного лазерного сплавления титанового порошка марки Ti-6Al-4V на установке EOSINT 280 при параметрах, установленных производителем для порошка данной марки и толщине слоя 30 мкм в среде аргона при давлении 5,0 атм. Фракционный состав порошка характеризовался следующими значениями диаметра сферических частиц: D-values D10 1/4 20 мкм; D50 1/4 35 мкм; D90 1/4 
45 мкм. Все образцы были получены в одной сессии СЛС без использования поддержек с применением кросс-штриховки с углом поворота между слоями $68^{\circ}$.

Образцы с ячеистой структурой были обработаны на электроискровом станке для придания параллельности сторон. Образцы имели размер $20 \times 20 \times 20$ мм и состояли из $4 \times 4 \times 4$ элементарных ячеек.

\section{3. Методика эксперимента}

Статическое нагружение образов по схеме одноосного сжатия выполнялось на электромеханической испытательной машине INSTRON 3382 со скоростью перемещения захвата 1,8 мм/мин. В ходе нагружения фиксировались перемещение подвижного захвата и усилие, а также выполнялась видеозапись одной из боковых поверхностей. Условия испытаний и анализируемые механические свойства установлены ISO 13314.

Циклическое нагружение призматических образцов размером 20×20×20 мм проведено на высокочастотной резонансной испытательной машине MIKROTRON 20 kN (RUMUL) при синусоидальном режиме приложения нагрузки по схеме одноосного «сжатия-сжатия» с коэффициентом асимметрии цикла $\mathrm{R}=0,1$. Испытания проведены в условиях мягкого нагружения с контролем по нагрузке. Максимальное усилие цикла составляло $\mathrm{F}_{\max }=1,6 \mathrm{\kappa H}$, что соответствовало максимальному условному напряжению в цикле 4 МПа. В ходе эксперимента контролировались изменения резонансной частоты колебания, величина перемещения подвижного захвата и количество циклов нагружения. В обоих случаях циклического и статического нагружения выполнялось наблюдение характера разрушения объекта оптическим методом.

Исследуемые образцы испытывались в двух направлениях. Осевое нагружение прикладывалось в направлении печати образцов (BD) и под углом $90^{\circ}$ к направлению печати.

Микроструктурные и фрактографические исследования были проведены на двухлучевом электронно-ионном микроскопе ThermoFischer Scios II LowVac.

\section{4. Результаты и обсуждение}

Для нижней поверхности балки характерно провисание металла под собственным весом в процессе СЛС, что является особенностью в формировании геометрии образцов с ячеистой архитектурой. Верхняя поверхность отличается меньшей кривизной благодара тому, что ранее кристаллизовавшиеся слои металла выступают поддержкой. В структуре наблюдается $\alpha^{\prime}$-мартенсит игольчатой морфологии вследствие высокой скорости охлажения, обусловленной малым объемом ванны расплава и высокой скоростью теплоотвода в ранее кристаллизовавшиеся слои. Исходное $\beta$-зерно либо имеет равноосную форму, либо вытянуто вдоль направления синтеза в результате эпитаксиального роста. Относительно крупные поры (10-40 мкм) в основном располагаются по границам ванны расплава, по-видимому в результате объемного эффекта при кристаллизации металла или недостаточной глубины проплавления (рис. 2 a). Газовая пористость, представленная более мелкими порами (единицы мкм), имеет более равномерное распределение (рис. 2 б). 


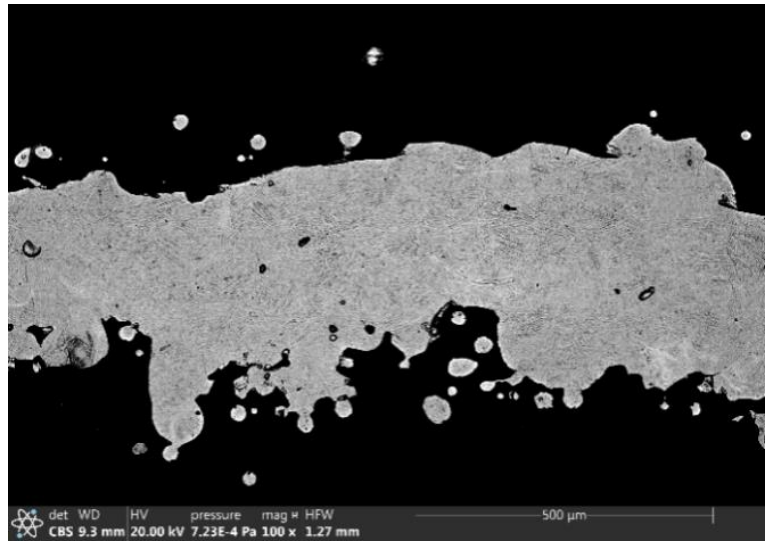

$a$

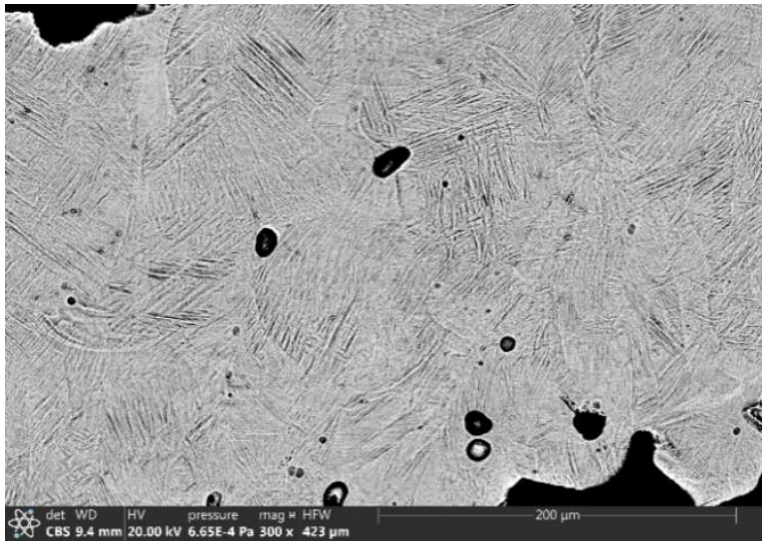

$\sigma$

Рис. 2. Структура балки ячеистой структуры (направление синтеза вверх)

Согласно результатам механических испытаний установлено, что диаграмма сжатия демонстрирует характерное для пористых (>50 \% пор) материалов поведение (рис. 3) [21]. Запись изображений боковой поверхности образцов как в ходе статического, так и при циклическом нагружении позволила проследить процесс разрушения и выявить закономерности в разрушении элементов архитектуры исследуемого материала (рис. 4 и 6). В условиях статического нагружения за участком упругой деформации (рис. 4 a) следует резкое падение нагрузки, связанное с выходом из строя первого сегмента ячеистой структуры (рис. 4 б). Далее следует участок плато, напряжения на котором изменяются скачкообразно в результате пластической деформации и «схлопывания» отдельных ячеек (рис. 4 в), однако среднее напряжение на этом участке меняется незначительно. На заключительном этапе после закрытия всех пор реализуется деформационное упрочнение монолитного материала, сопровождающееся ростом напряжения (рис. 4 г).

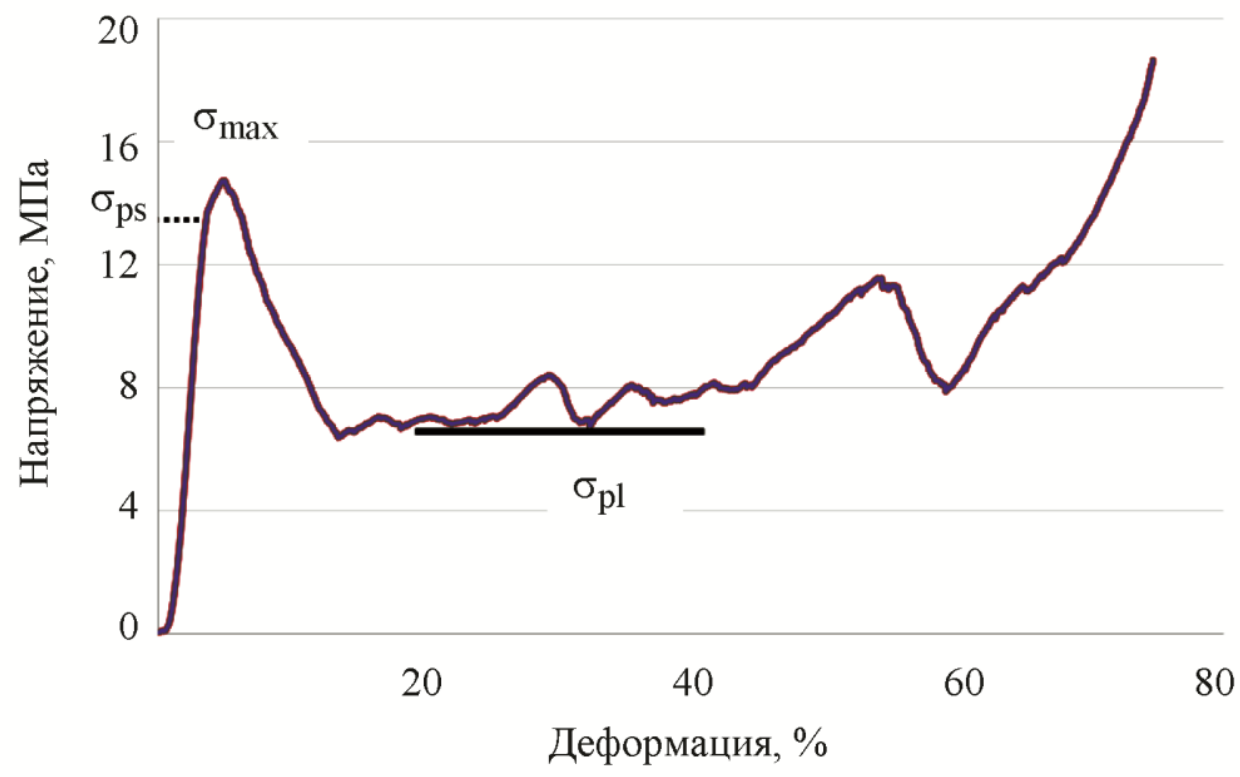

Рис. 3. Диаграмма статического сжатия 


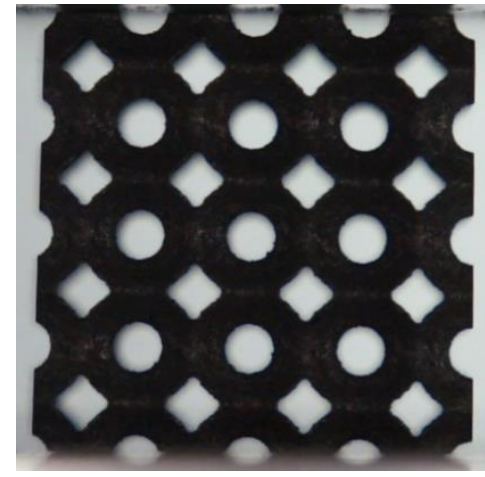

$a$

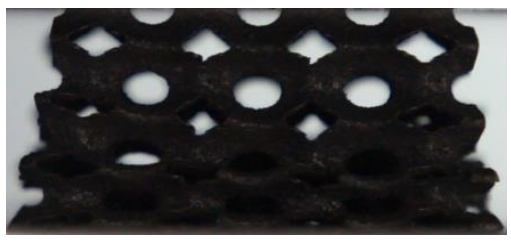

B

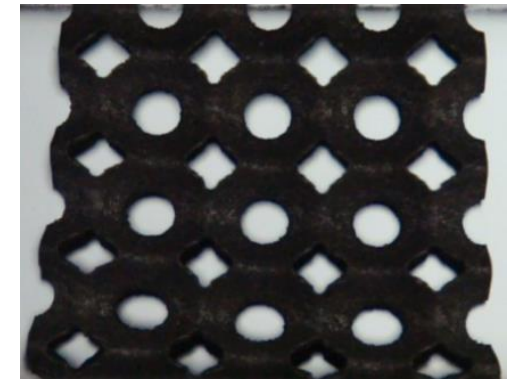

$\sigma$

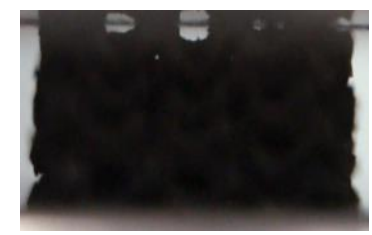

Рис. 4. Общий вид образца на различных стадиях статического сжатия: $a$ - стадия $1 ; \sigma$ - стадия $2 ; в$ - стадия $3 ;$ г - стадия 4

По результатам предварительных испытаний установлено, что уровень напряжения, при котором происходит усталостное разрушение образца на базе $\approx 2 \times 10^{5}$ циклов, составляет примерно 0,3 от $\sigma_{\mathrm{ps}}$ (proof strength).

Как уже отмечалось, в зависимости от направления печати материал образца обладает выраженной анизотропией свойств и, как следствие, различным сопротивлением распространению трещин [22]. Кроме того, для материалов с ячеистой архитектурой, изготовленных методом СЛС, характерно нарушение формы, связанное с появлением овальности при печати сфероидальных элементов (рис. 1 в). Учет указанных особенностей при проектировании и изготовлении ответственных конструктивных элементов является важной прикладной инженерной задачей.

Типичное поведение ячеистого материала в условиях циклического нагружения отражено на графиках (рис. 5). Так, в исходном состоянии размах деформации в результате приложения нагрузки одинаков независимо от ориентации образца. Общее количество циклов до разрушения $3 \times 10^{5}$ и $1,4 \times 10^{5}$, т. е. отличается более чем в два раза при сохранении идентичной жесткости и без потери устойчивости.

В качестве информативного параметра, учитывающего потерю устойчивости, было выбрано положение траверсы, задающей среднее усилие. После сжатия образца на 0,1 мм эксперимент останавливался. При этом визуально наблюдались множественные растрескивания. Несмотря на это после полной разгрузки образец принимал свою исходную форму, а его несущая способность снижалась незначительно, т. е. объект находился в состоянии, предшествующем его катастрофическому разрушению.

На графике рис. 5 представлены совмещенные диаграммы статического перемещения траверсы (задает среднее усилие цикла), из которых видно, что начало потери устойчивости образцов, ориентированных вдоль параллельного направления приложения усилия в направлении BD происходит после $2 \times 10^{5}$ циклов нагружения. В то время как при ориентации «направление приложения усилия перпендикулярно направление печати BD» начало потери устойчивости начинается до достижения $2 \times 10^{4}$ циклов нагружения. 


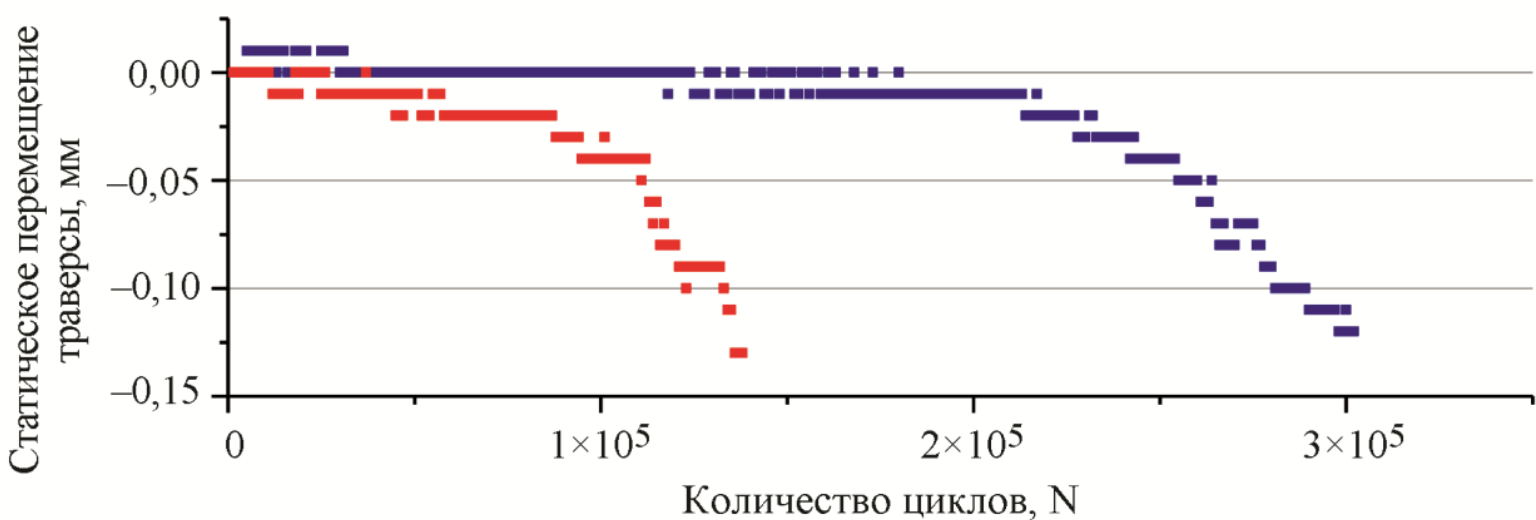

Рис. 5. Диаграммы циклического нагружения ячеистых образцов в направлении плоскости $\mathrm{BD}(-)$ и перпендикулярно BD (-)

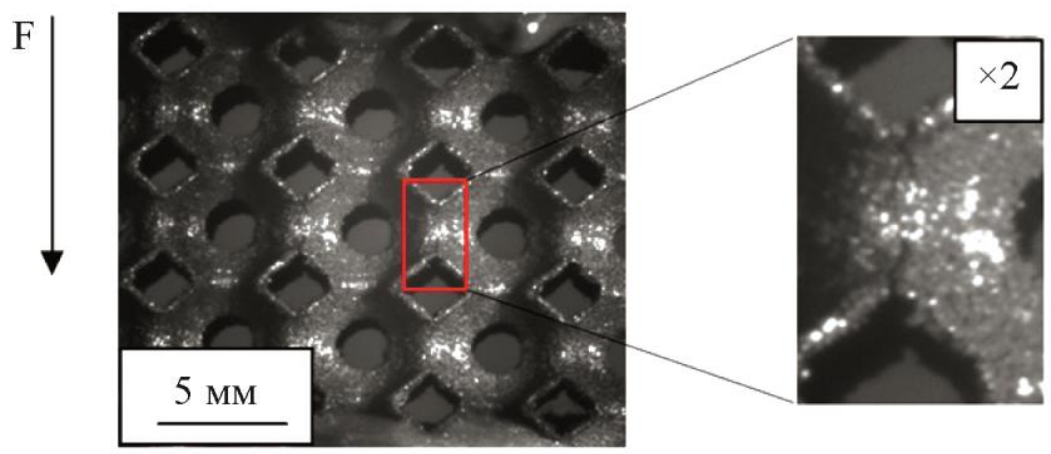

$a$

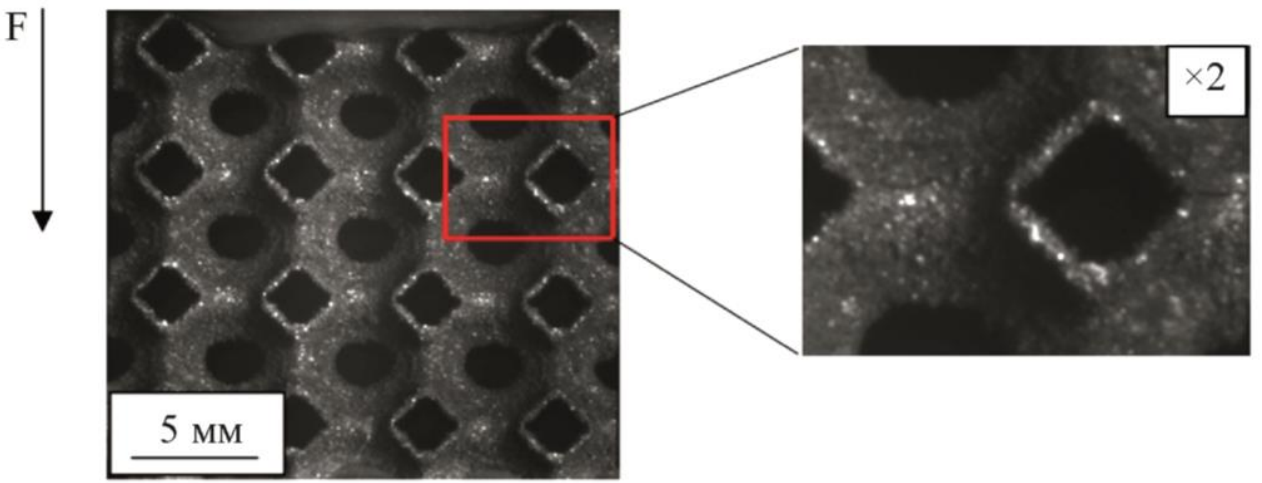

$\sigma$

Рис. 6. Боковая поверхность ячеистого образца при различной ориентации относительно оси приложения циклического нагружения: $a$ - ориентировка XO-BD; $\sigma$ - ориентировка $\mathrm{BD}$

Из анализа оптических изображений (рис. 6), сделанных в ходе эксперимента, установлено, что в ходе усталостного нагружения при ориентации плоскости печати образцов вдоль оси нагружения и напряжениях, составляющих $\sigma_{\max }=0,3 \sigma_{\mathrm{ps}}$, первые признаки разрушения возникли при $\mathrm{N}=1,23 \times 10^{5}$ циклов. Разрушение происходило преимущественно в плоскости печати. При циклическом нагружении в направлении, перпендикулярном печати, траектория распространения магистральной трещины также совпадала с плоскостью печати. В обоих случаях разрушение ячеистых образцов реализовывалось поэтапным разрушением отдельных сегментов. Выявленной особенностью усталостного разрушения ячеистого образца является то, что при нагружении в направлении, перпендикулярном плоскости печати, 
максимальное количество циклов составило $\mathrm{N}=3 \times 10^{5}$, что более чем в два раза превышает количество циклов нагружения для образца при его ориентации, реализующей нагружение вдоль плоскости печати.

Таким образом, возможная причина увеличения долговечности объекта при его ориентации, реализующей циклическое нагружение вдоль направления $\mathrm{BD}$, это выявленная особенность механизма разрушения материалов со сложной архитектурой - наличие двух конкурирующих процессов: разрушения в главной плоскости в результате приложения циклических нагрузок и разрушение отдельных элементов, связанное с особенностью синтеза исследуемого класса материалов. Предположение о протекании конкурирующих процессов разрушения в направлении BD подтверждается результатами фрактографического анализа (рис. 7). При нагружении образцов в направлении, перпендикулярном плоскости BD, рельеф сглаженный, характерный для усталостного разрушения. Разделение сегментов произошло в одной главной плоскости (рис. 7 a).

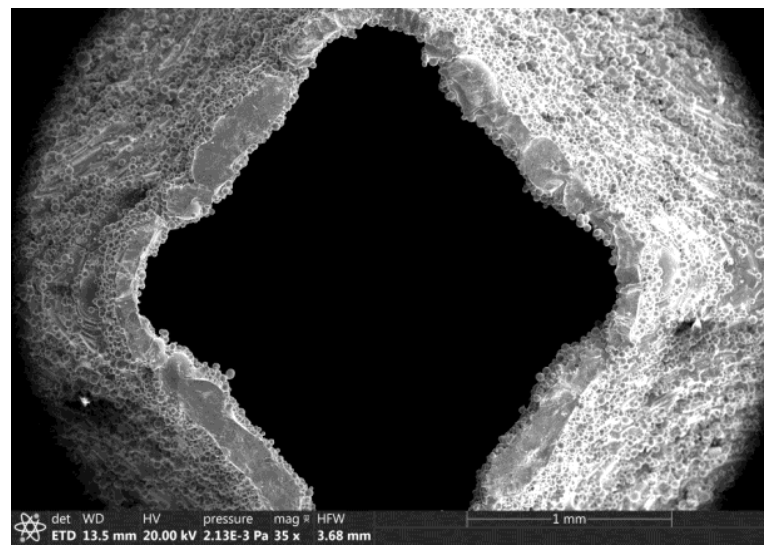

$a$

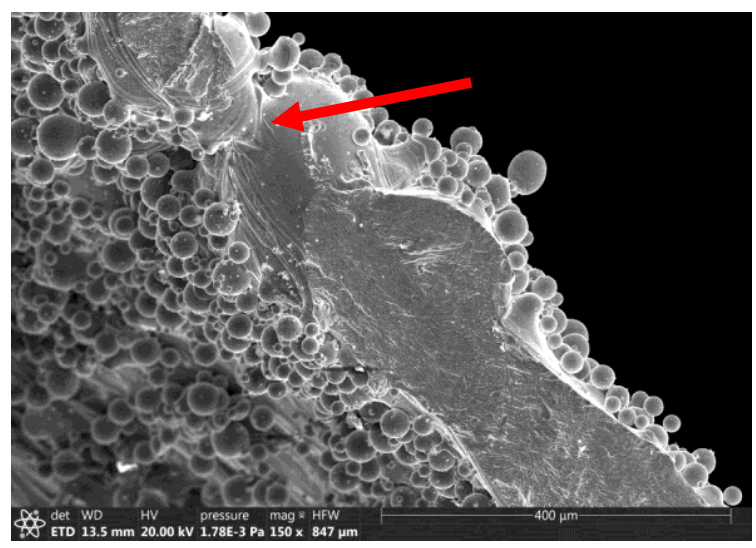

$\sigma$

Рис. 7. Поверхность разрушения образца при нагружении перпендикулярно напрвлению BD

Несмотря на наличие зон несплавления (рис. 7 б, указано стрелкой) вторичных трещин не наблюдается, т. е. локальные несплошности в виде непроплавов не являются концентраторами, вызывающими формирование вторичных трещин. В то же время наблюдаемые на изломах непроплавы являются «плоскими порами», вытянутыми поперек направления сплавления, в результате разрушение по этой плоскости облегчено.

В случае приложения циклических нагрузок вдоль направления BD магистральная трещина также распространяется и в перпендикулярной плоскости (рис. 8).

При большем увеличении наблюдается множественное растрескивание ортогонально к плоскости распространения магистральной трещины (рис. 9). Как и в случае нагружения образца в направлении, перпендикулярном BD, распространение трещин в большинстве случаев происходит в объеме материала, т. е. участки зон сплавления отдельных частиц порошка в данном случае не являются концентраторами. А трещины на боковой поверхности распространяются вдоль границ структурных элементов. 

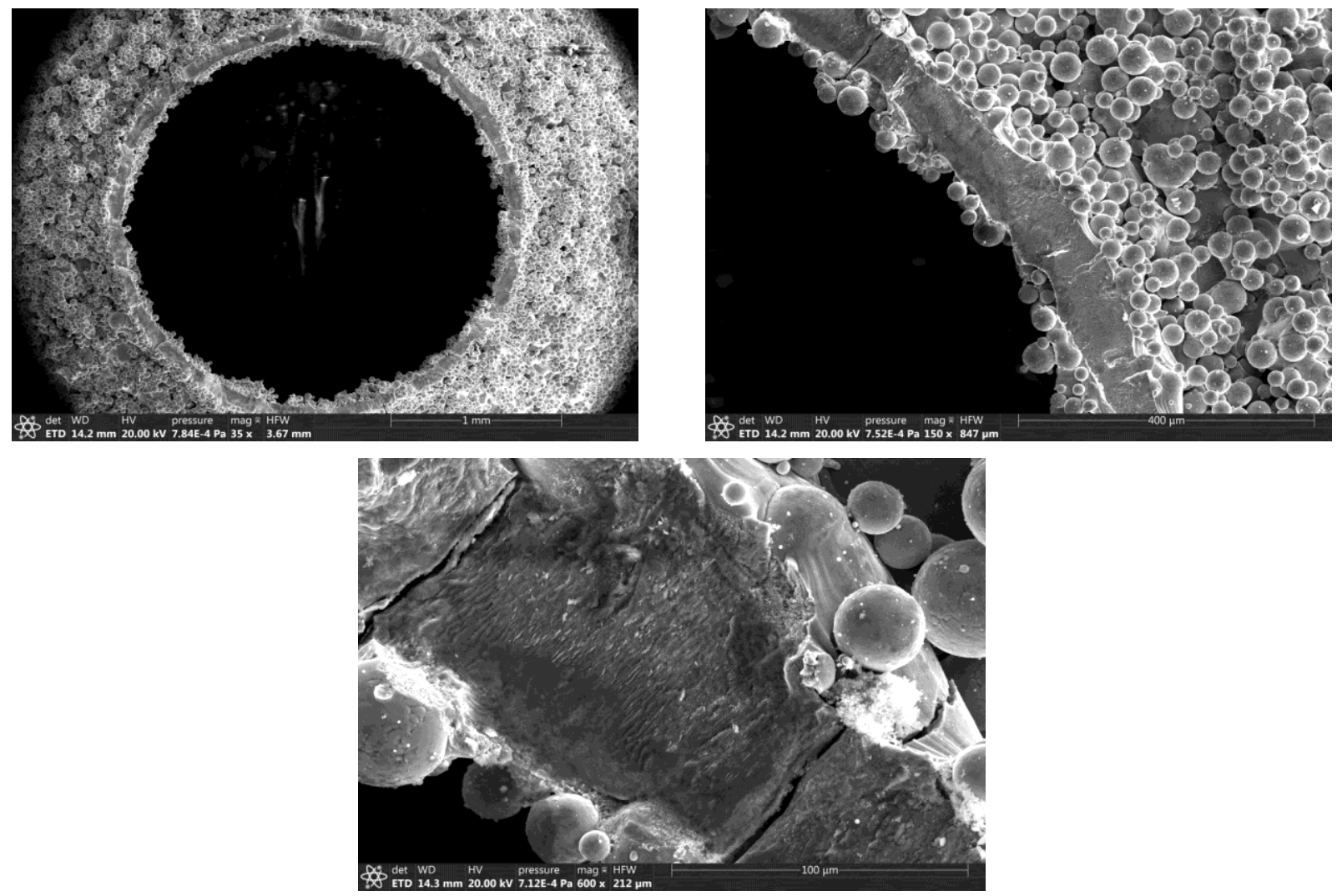

Рис. 8. Фрактограммы поверхности разрушения ячеистого образца при ориентации BD вдоль оси приложения нагрузки

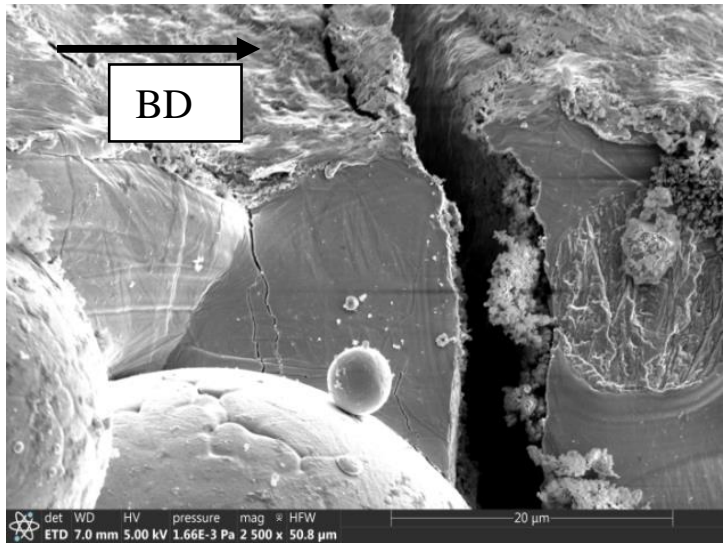

$a$

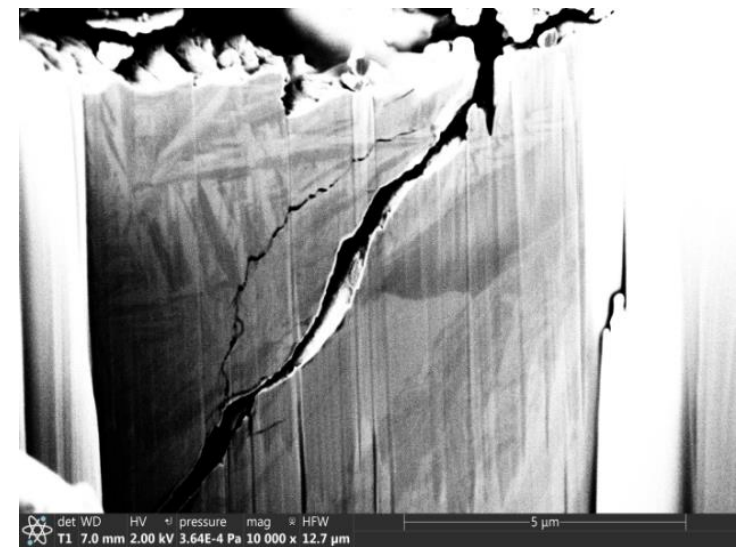

$\sigma$

Рис. 9. Боковая поверхность стенки: $a$ - РЭМ; $\sigma$ - ионное травление

Таким образом установлено, что причиной увеличения долговечности объекта при его ориентации, реализующей циклическое нагружение вдоль направления BD, является выявленная особенность механизма разрушения. Так, при данной ориентации в условиях циклического нагружения при усталостном разрушении наблюдаются два конкурирующих процесса: разрушение отдельных сегментов по плоскости печати и формирование вторичных трещин в перпендикулярном направлении, блокирующих распространение магистральной. Стоит отметить, что в условиях статического нагружения подобного эффекта не наблюдается. 


\section{5. Заключение}

Продемонстрировано влияние направления синтеза объектов ячеистой формы, полученных лазерным селективным сплавлением, на характеристики разрушения в условиях циклического нагружения. Установлено, что при ориентации образца в направлении приложения усилия, совпадающем с направлением синтеза, усталостное разрушение носит сложный характер, обеспечивающий тем самым повышенное сопротивление усталостному разрушению. Выявленная особенность подчеркивает важность выбора ориентации модели для лазерной 3D-печати для рационального проектирования изделий.

\section{Благодарности}

Работа поддержана в рамках мероприятия «Создание и функционирование сети международных научно-методических центров для распространения лучиих международных практик подготовки, переподготовки и стажировки продвинутых кадров цифрровой экономики в областях математики, информатики, технологий» (Соглашение № 075-152019-1907 от 09.12.2019) и в соответствии с планом работ ИМАШ УрО РАН по теме АААА-А18-118020790145-0 в части определения характеристик сопротивления усталостному разрушению. Экспериментальная часть работы выполнена с использованием оборудования ЦКП «Пластометрия» ИМАШ УрО РАН.

\section{Литература}

1. Investigation of the structure and mechanical properties of additively manufactured Ti-6Al-4V biomedical scaffolds designed with a Schwartz primitive unit-cell / N. Soro, H. Attar, X. Wu, M. S. Dargusch // Materials Science and Engineering A. - 2019. - A 745. - P. 195-202. DOI: $10.1016 /$ j.msea.2018.12.104.

2. Golodnov A. I., Loginov Y. N., Stepanov S. I. Numeric loading simulation of titanium implant manufactured using 3d printing // Solid State Phenomena. - 2018. - Vol. 284 SSP. - P. 380-385. DOI: $10.4028 /$ www.scientific.net/SSP.284.380.

3. Design and 3D-printing of titanium bone implants: brief review of approach and clinical cases / V. V. Popov, G. Muller-Kamskii, A. Kovalevsky, A. Kolomiets, J. Ramon // Biomedical Engineering Letters. - 2018. - Vol. 8 (4). - P. 337-344. - DOI: 10.1007/s13534-018-0080-5.

4. Functionality-packed additively manufactured porous titanium implants / I. A. J. Van Hengel, F. S. A. Gelderman, S. Athanasiadis, M. Minneboo, H. Weinans, A. C. Fluit, B. C. J. Van der Eerden, L. E. Fratila-Apachitei, I. Apachitei, A. A. Zadpoor // Materials Today Bio. - 2020. Vol. 7. - DOI: 10.1016/j.mtbio.2020.100060.

5. De Jonge C. P., Kolken H. M. A., Zadpoor A. A. Non-Auxetic Mechanical Metamaterials // Materials. - 2019. - Vol. 12 (4). - P. 635 (1-21). - DOI: 10.3390/ma12040635.

6. Килина П. Н., Дроздов А. А., Сиротенко Л. Д. Формирование образцов с ячеистой структурой методом селективного лазерного спекания металлических порошков // Металлообработка. - 2015. - № 3 (87). - С. 29-31.

7. Mechanical properties of diamond lattice Ti-6Al-4V structures produced by laser powder bed fusion: on the effect of the load direction / A. Cutolo, B. Engelen, W. Desmet, B. Van Hooreweder // Journal of the Mechanical Behavior of Biomedical Materials. - 2020. - Vol. 104. - P. 103656 (1-15). - DOI: 10.1016/j.jmbbm.2020.103656.

8. Effect of Built Geometry on the Microstructure and Strength Characteristics of the Ti-6Al-4V. Alloy Prepared by the Selective Laser Melting / N. V. Kazantseva, I. V. Ezhov, N. I. Vinogradova, M. V. Il'inykh, A. S. Fefelov, D. I. Davydov, O. A. Oleneva \& M. S. Karabanalov // Phys. Metals Metallogr. - 2018. - No. 119. - P. 1079-1086. - DOI: 10.1134/S0031918X18110066. 
9. Crack propagation and fracture toughness of Ti6Al4V alloy produced by selective laser melting / V. Cain, L. Thijs, J. Van Humbeeck, B. Van Hooreweder, R. Knutsen // Additive Manufacturing. - 2015. - Vol. 5 - P. 68-76. - DOI: 10.1016/j.addma.2014.12.006.

10. Anisotropy and heterogeneity of microstructure and mechanical properties in metal additive manufacturing: A critical review / Y. Kok, X. P. Tan, P. Wang, M. L. S. Nai, N. H. Loh, E. Liu, S. B. Tor // Materials and Design. - 2018. - Vol. 139. - P. 565-586. - DOI: 10.1016/j.matdes.2017.11.021. URL: https://www.sciencedirect.com/science/article/pii/S0264127517310493\#!

11. Hartunian P., Eshraghi M. Effect of Build Orientation on the Microstructure and Mechanical Properties of Selective Laser-Melted Ti-6Al-4V Alloy // J. Manuf. Mater. Process. - 2018. -2 (4). P. 69. - DOI: 10.3390/jmmp2040069.

12. On the size and orientation effect in additive manufactured Ti-6Al-4V / D. Barba, C. Alabort, Y. T. Tang, M. J. Viscasillas, R. C. Reed, E. Alabort // Materials and Design. - 2020. Vol. 186. - P. 108235. - DOI: 10.1016/j.matdes.2019.108235.

13. Effect of Annealing on the Structure and Properties of Titanium Alloy with Cellular Architecture for Medical Applications / S. I. Stepanov, Yu. N. Loginov, V. P. Kuznetsov, A. A. Popov // Metal Science and Heat Treatment. - 2018. - Vol. 60 (5-6). - P. 315-321. - DOI: 10.1007/s11041018-0278-2.

14. Relationship between unit cell type and porosity and the fatigue behavior of selective laser melted metabiomaterials / S. Amin Yavari, S. M. Ahmadi, R. Wauthle, B. Pouran, J. Schrooten, H. Weinans, A. A. Zadpoor // Journal of the Mechanical Behavior of Biomedical Materials. - 2015. Vol. 43. - P. 91-100. - DOI: 10.1016/j.jmbbm.2014.12.015.

15. Van Hooreweder B., Apers Y., Lietaert K., Kruth J.P. Improving the fatigue performance of porous metallic biomaterials produced by selective laser melting // Acta Biomaterialia. - 2017. Vol. 47. - P. 193- 202. - DOI: 10.1016/j.actbio.2016.10.005.

16. Ботвина Л. Р. Кинетика разрушения конструкционных материалов. - М. : Наука, 1989. $230 \mathrm{c}$.

17. Коцаньда С. Усталостное растрескивание металлов / пер. с польского Г. Н. Мехеда; под ред. С. Я. Яремы. - М. : Металлургия, 1990. - 632 с.

18. Volkov S. S. The Effect of Damage at An Ensemble of Microstructure Points on the Margin of Safety in Structurally Heterogeneous Materials // Diagnostics, Resource and Mechanics of materials and structures. - 2019. - Iss. 5. - P. 60-72. - DOI: 10.17804/2410-9908.2019.5.060-072 . URL: http://dream-journal.org/issues/2019-5/2019-5_274.html (accessed: 13.08.2020).

19. Loginov Y., Stepanov S., Khanykova C. Inhomogeneity of deformed state during compression testing of titanium implant // 2017 MATEC Web of Conferences, 13th International ScientificTechnical Conference on Dynamic of Technical Systems, DTS 2017, Rostov-on-Don, Sept. 13-15, 2017. - EDP Sciences, 2017. - P. 03009. - Vol. 132. - DOI: 10.1051/matecconf/201713203009.

20. Effect of Selective Laser Melting Process Parameters on the Quality of Al Alloy Parts: Powder Characterization, Density, Surface Roughness, and Dimensional Accuracy / A. H. Maamoun, Y. F. Xue, M. A. Elbestawi, S. C. Veldhuis // Materials. - 2018. - Vol. 11 (12). - P. 2343. DOI: $10.3390 / \mathrm{ma1} 1122343$.

21. Gibson L., \& Ashby M. Cellular Solids: Structure and Properties. - 2nd ed. Cambridge Solid State Science Series. - Cambridge : Cambridge University Press, 1997. DOI: $10.1017 / \mathrm{CBO} 9781139878326$.

22. $\mathrm{Xu} \mathrm{Z}$. W., Liu A., Wang X. S. The influence of building direction on the fatigue crack propagation behavior of Ti6Al4V alloy produced by selective laser melting // Materials Science and Engineering: A. - 2019. - Vol. 767. - P. 138409. - DOI: 10.1016/j.msea.2019.138409. 